\title{
Variação das dimensões características e da forma dos frutos de café durante o processo de secagem
}

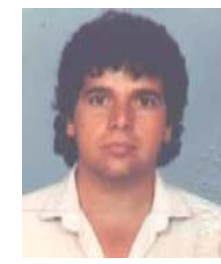

Paulo C. Corrêa' ${ }^{1}$ Paulo C. Afonso Júnior², Daniel M. de Queiroz³, Cristiane P. Sampaio ${ }^{4} \&$ Janayna B. Cardoso ${ }^{5}$

\author{
1 DEA/UFV. CEP 36.571-000, Viçosa, MG. Fone: (31) 3891-2270. E-mail: copace@mail.ufv.br (Foto) \\ 2 DEA/UFV. Bolsista FAPEMIG. E-mail: pjunior@alunos.ufv.br \\ 3 DEA/UFV. E-mail: queiroz@mail.ufv.br \\ ${ }^{4}$ DEA/UFV. E-mail: csampaio@alunos.ufv.br \\ ${ }^{5}$ DTA/UFV. Bolsista Iniciação Científica
}

Protocolo $16-19 / 2 / 2002$

\begin{abstract}
Resumo: O presente trabalho teve como objetivo avaliar o efeito da variação do teor de umidade nas dimensões e propriedades físicas-esfericidade e circularidade do fruto do café das espécies arabica e canephora, de diferentes variedades. Os resultados obtidos permitiram afirmar que a redução do teor de umidade afeta de forma acentuada as características físicas do fruto do cafeeiro das espécies e variedades estudadas, indicando que são importantes as variações das dimensões do fruto ao longo do processo de secagem e, ainda, que as expressões matemáticas ajustadas aos dados obtidos representaram de forma satisfatória os fenômenos estudados.
\end{abstract}

Palavras-chave: café, teor de umidade, propriedades físicas

\section{Variation of characteristic dimensions and forms of coffee fruits during drying process}

\begin{abstract}
The present work had as objective to evaluate the effect of moisture content variation on dimensions and physical properties (sphericity and roundness) of coffee fruit of the arabica and canephora species, for different varieties. The obtained results show that the reduction of moisture content affects in an accentuated way the physical characteristics of coffee fruit of the studied species and varieties, indicating that the variations of dimensions of the fruit along the drying process are important. Further, the mathematical expressions adjusted to the data were satisfactory for the studied phenomena.
\end{abstract}

Key words: coffee, moisture content, physical properties

\section{INTRODUÇÃO}

Informações concernentes à forma e ao tamanho, entre outras características físicas dos produtos agrícolas, são consideradas de grande importância para estudos envolvendo transferência de calor e massa e movimentação de ar em produtos granulares. Outras aplicações significativas do conhecimento das características dimensionais de frutos e grãos estão associadas à elaboração de projetos de unidades de processamento e ao dimensionamento de equipamentos de secagem, separação, armazenagem e classificação (Weber, 1995).

A secagem de produtos agrícolas com alto teor de umidade inicial, como os frutos do café, é acompanhada de uma significativa redução de volume, pelo qual o produto sofre alterações em suas dimensões e forma originais. Ratti (1994) e Zogzas et al. (1994) observaram que o encolhimento de produtos vegetais durante a secagem não é função exclusiva do teor de umidade, mas também, das condições do processo e da geometria do produto, uma vez que materiais biológicos porosos quando desidratados contraem-se diferentemente nas direções longitudinal, tangencial e radial (Fortes \& Okos, 1980; Cavalcanti Mata et al., 1986).

Os grãos e frutos, de modo geral, não apresentam um formato geométrico perfeitamente definido, tornando necessário para a solução de problemas relacionados à sua geometria, assumir para o produto uma forma conhecida, o que acarreta em aproximações e possíveis erros. Agrawal et al. (1972) observaram que, para maioria dos produtos agrícolas, muitas dessas soluções são obtidas assumindo-se, as formas geométricas de um esferóide ou elipsóide composto por três dimensões características, que são os eixos maior, médio e menor. Entretanto, Curray citado por Mohsenin (1986), com o intuito de analisar esses desvios propôs alguns métodos para determinar a esfericidade, ou seja, o grau de aproximação da forma do produto com a esfera, e a circularidade, que indica o 
quanto se aproxima a área da projeção do material na posição de repouso de um círculo, de acordo com as seguintes expressões:

$$
\begin{gathered}
\mathrm{E}=\left[\frac{\mathrm{V}_{\mathrm{s}}}{\mathrm{V}_{\mathrm{ec}}}\right]^{1 / 3} \\
=\left[\frac{\left.\left.\left(\frac{\pi \mathrm{abc}}{6}\right)\right]^{1 / 3}=\frac{(\mathrm{abc})^{1 / 3}}{\mathrm{a}}\right)}{\mathrm{C}=\frac{\mathrm{A}_{\mathrm{p}}}{\mathrm{A}_{\mathrm{c}}}}\right.
\end{gathered}
$$

em que:

E - esfericidade, adimensional

C - circularidade, adimensional

$\mathrm{V}_{\mathrm{s}} \quad$ - volume do sólido, $\mathrm{mm}^{3}$

$\mathrm{V}_{\mathrm{ec}}^{\mathrm{s}}$ - volume da esfera circunscrita, $\mathrm{mm}^{3}$

$\mathrm{A}_{\mathrm{p}}$ - área projetada do fruto na posição de repouso, $\mathrm{mm}^{2}$

$\mathrm{A}_{\mathrm{c}}$ - área do menor círculo circunscrito, $\mathrm{mm}^{2}$

$\mathrm{a}, \mathrm{b}, \mathrm{c}$ - eixos ortogonais do produto, $\mathrm{mm}$

Soares (1986), empregou para soja as aproximações de um esferóide e elipsóide, para estimar o volume dos grãos na faixa de umidade de 10,7 a 23,7\% base úmida e verificou, para ambas aproximações, uma tendência de superestimar os resultados, quando comparados com os valores de volume determinados pelo método de complementação de volume utilizando tolueno. Em média os resultados obtidos pelas aproximações apresentaram erros próximos a 1,2\%. O mesmo autor e outros estudos que envolvem a forma geométrica de produtos agrícolas, têm empregado o conceito da esfera de volume equivalente ao do produto estudado (Almeida, 1979; Soares, 1986).

Mudanças das características dimensionais dos produtos, devido à sua desidratação, são relatadas como as primeiras causas das alterações das principais propriedades físicas de produtos agrícolas (Bala \& Woods, 1984; Ratti, 1994; Sokhansanj \& Lang, 1996). Para frutos de café, não se encontram, na literatura especializada, resultados de estudos relacionados à variação do tamanho e da forma em função do seu processamento.

Diante o exposto, o presente trabalho teve como objetivo avaliar o efeito do teor de umidade na variação das dimensões características e propriedades físicas esfericidade e circularidade dos frutos do café de diferentes espécies e variedades.

\section{MATERIAL E MÉTODOS}

O presente trabalho foi realizado no Laboratório de Propriedades Físicas e Qualidade de Produtos Agrícolas pertencente ao CENTREINAR, localizado na Universidade Federal de Viçosa, Viçosa, MG.
Foram utilizados frutos de café das variedades da espécie Coffea arabica, variedades: Catuaí Vermelho, Catuaí Amarelo, Mundo Novo, Catimor e da espécie Coffea canephora o Conilon. Colhidos no estádio cereja, homogeneizados e posteriormente selecionados e identificados, para cada variedade, 10 frutos sadios e intactos foram submetidos à secagem em estufa com ventilação forçada com temperatura do ar de aproximadamente $35 \pm 3{ }^{\circ} \mathrm{C}$, obtendo-se 10 diferentes níveis de teor de umidade.

O acompanhamento da redução do teor de umidade com o decorrer do tempo de secagem foi realizada por meio gravimétrico (perda de peso), conhecendo-se o teor de umidade inicial dos frutos. Para este monitoramento foi utilizada uma balança analítica com precisão de $0,01 \mathrm{~g}$.

Os teores de umidade inicial dos produtos foram determinados pelo método da estufa, $105 \pm 3{ }^{\circ} \mathrm{C}$, durante $24 \mathrm{~h}$, em três repetições (Brasil, 1992).

Periodicamente, durante o processo de secagem, os frutos do café eram retirados da estufa e encaminhados para determinação de suas dimensões características, a partir da medição de seus três eixos ortogonais com paquímetro digital, assumindo-se a forma de um esferóide tri-axial oblato para o fruto do café (Fig. 1).

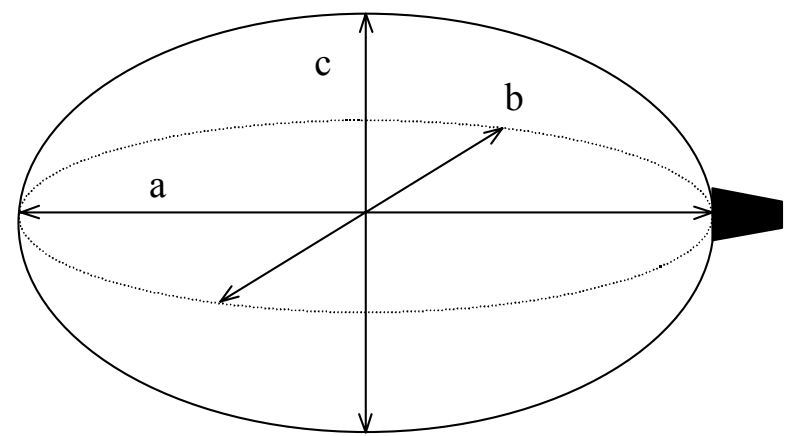

Figura 1. Desenho esquemático de um fruto de café considerado como um esferóide tri-axial oblato e suas dimensões características

A forma dos frutos do cafeeiro foi analisada pela esfericidade e circularidade, que foram calculadas a partir da determinação dos eixos ortogonais empregando-se as Eqs. 1 e 2, respectivamente.

Os dados foram submetidos à análise de regressão com finalidade de selecionar o modelo matemático mais adequado para expressar a relação entre o teor de umidade e as variáveis estudadas. Para o ajuste dos modelos matemáticos aos dados experimentais, utilizando-se os programas computacionais TableCurve 2D® (versão 2.12) e STATISTICA® (versão 5.0), estimaram-se os valores dos parâmetros do modelo em função da variável independente teor de umidade do produto.

\section{RESULTADOS E DISCUSSÃO}

$\mathrm{Na}$ Tabela 1 estão apresentados os valores das dimensões características do fruto do café, eixos ortogonais maior (a), médio (b) e menor (c), os índices de contração de cada eixo em relação a sua dimensão original e os valores calculados de esfericidade e circularidade. 
Tabela 1. Dimensões médias dos eixos (a, b e c) ortogonais do fruto do café e os respectivos desvios padrões (dp), índices de redução, esfericidade (E) e circularidade (C) para diferentes variedades

\begin{tabular}{|c|c|c|c|c|c|c|c|c|c|c|c|}
\hline \multirow{2}{*}{$\begin{array}{l}\text { Umidade } \\
\text { (b. s.) }\end{array}$} & \multicolumn{6}{|c|}{ Eixos Ortogonais (mm) } & \multirow{2}{*}{$\begin{array}{l}\mathrm{a} / \mathrm{a}_{0} \\
(\%)\end{array}$} & \multirow{2}{*}{$\begin{array}{l}\mathrm{b} / \mathrm{b}_{0} \\
(\%)\end{array}$} & \multirow{2}{*}{$\begin{array}{l}\mathrm{c} / \mathrm{c}_{0} \\
(\%)\end{array}$} & \multirow{2}{*}{$\begin{array}{c}\mathrm{E} \\
(\%)\end{array}$} & \multirow{2}{*}{$\begin{array}{c}\mathrm{C} \\
(\%)\end{array}$} \\
\hline & a & $\mathrm{dp}$ & $\mathrm{b}$ & $\mathrm{dp}$ & $\mathrm{c}$ & $d p$ & & & & & \\
\hline \multicolumn{12}{|c|}{ Catuaí Vermelho } \\
\hline 2,03 & 14,99 & 0,64 & 11,97 & 0,60 & 13,08 & 0,57 & 100,00 & 100,00 & 100,00 & 88,65 & 87,26 \\
\hline 1,63 & 13,59 & 0,62 & 10,56 & 0,51 & 12,12 & 0,60 & 90,66 & 88,22 & 92,66 & 88,49 & 89,18 \\
\hline 1,31 & 13,52 & 0,69 & 10,09 & 0,72 & 11,45 & 0,42 & 90,19 & 84,29 & 87,54 & 85,82 & 84,69 \\
\hline 0,85 & 13,22 & 0,69 & 9,40 & 0,60 & 10,64 & 0,47 & 88,19 & 78,53 & 81,35 & 83,02 & 80,48 \\
\hline 0,48 & 13,23 & 0,78 & 9,26 & 0,60 & 10,44 & 0,53 & 88,26 & 77,36 & 79,82 & 82,05 & 78,91 \\
\hline 0,42 & 13,17 & 0,68 & 9,23 & 0,60 & 10,47 & 0,25 & 87,86 & 77,11 & 80,05 & 82,29 & 79,50 \\
\hline 0,36 & 13,16 & 0,75 & 9,26 & 0,69 & 10,43 & 0,35 & 87,79 & 77,36 & 79,74 & 82,31 & 79,26 \\
\hline 0,20 & 12,99 & 0,67 & 9,24 & 0,63 & 10,36 & 0,33 & 86,66 & 77,19 & 79,20 & 82,78 & 79,75 \\
\hline 0,14 & 12,94 & 0,64 & 9,11 & 0,52 & 10,12 & 0,38 & 86,32 & 76,11 & 77,37 & 81,96 & 78,21 \\
\hline 0,12 & 12,90 & 0,66 & 9,09 & 0,51 & 10,16 & 0,38 & 86,06 & 75,94 & 77,68 & 82,18 & 78,76 \\
\hline \multicolumn{12}{|c|}{ Catuaí Amarelo } \\
\hline 1,94 & 15,33 & 0,70 & 11,87 & 0,69 & 12,53 & 1,09 & 100,00 & 100,00 & 100,00 & 85,86 & 81,74 \\
\hline 1,50 & 13,95 & 0,64 & 10,47 & 0,88 & 11,33 & 0,69 & 91,00 & 88,21 & 90,42 & 84,79 & 81,22 \\
\hline 1,22 & 13,55 & 0,68 & 10,11 & 0,78 & 11,07 & 0,52 & 88,39 & 85,17 & 88,35 & 84,79 & 81,70 \\
\hline 0,92 & 13,76 & 0,73 & 9,90 & 0,70 & 10,81 & 0,59 & 89,76 & 83,40 & 86,27 & 82,68 & 78,56 \\
\hline 0,59 & 13,33 & 0,47 & 10,00 & 0,71 & 10,55 & 0,57 & 86,95 & 84,25 & 84,20 & 84,05 & 79,14 \\
\hline 0,52 & 13,36 & 0,71 & 10,01 & 0,68 & 10,50 & 0,53 & 87,15 & 84,33 & 83,80 & 83,82 & 78,59 \\
\hline 0,45 & 13,53 & 0,66 & 9,70 & 0,75 & 10,50 & 0,63 & 88,26 & 81,72 & 83,80 & 82,25 & 77,61 \\
\hline 0,26 & 13,38 & 0,37 & 9,53 & 0,62 & 10,36 & 0,32 & 87,28 & 80,29 & 82,68 & 82,01 & 77,43 \\
\hline 0,19 & 13,03 & 1,49 & 9,42 & 0,83 & 10,30 & 0,80 & 85,00 & 79,36 & 82,20 & 82,99 & 79,05 \\
\hline 0,11 & 13,00 & 1,48 & 9,39 & 0,88 & 10,28 & 0,79 & 84,80 & 79,11 & 82,04 & 82,97 & 79,08 \\
\hline \multicolumn{12}{|c|}{ Mundo Novo } \\
\hline 1,63 & 14,66 & 0,88 & 11,46 & 0,70 & 11,95 & 0,86 & 100,00 & 100,00 & 100,00 & 86,05 & 81,50 \\
\hline 1,34 & 14,56 & 0,92 & 10,98 & 0,58 & 11,66 & 0,96 & 99,32 & 95,81 & 97,57 & 84,51 & 80,07 \\
\hline 1,02 & 14,29 & 0,80 & 10,82 & 0,53 & 11,51 & 0,86 & 97,48 & 94,42 & 96,32 & 84,79 & 80,53 \\
\hline 0,58 & 14,39 & 0,48 & 9,70 & 0,58 & 11,31 & 0,76 & 98,16 & 84,64 & 94,64 & 80,93 & 78,60 \\
\hline 0,48 & 14,28 & 1,59 & 9,75 & 0,58 & 11,30 & 0,68 & 97,41 & 85,08 & 94,56 & 81,44 & 79,12 \\
\hline 0,37 & 14,29 & 0,82 & 9,83 & 0,45 & 11,19 & 0,63 & 97,48 & 85,78 & 93,64 & 81,35 & 78,28 \\
\hline 0,28 & 14,69 & 0,86 & 9,63 & 0,48 & 11,08 & 0,71 & 100,20 & 84,03 & 92,72 & 79,08 & 75,43 \\
\hline 0,23 & 14,59 & 1,10 & 9,65 & 0,64 & 11,10 & 0,71 & 99,52 & 84,21 & 92,89 & 79,52 & 76,05 \\
\hline 0,17 & 14,42 & 0,62 & 9,37 & 0,48 & 10,81 & 0,57 & 98,36 & 81,76 & 90,46 & 78,65 & 74,92 \\
\hline 0,12 & 14,28 & 0,56 & 9,30 & 0,56 & 10,78 & 0,49 & 97,41 & 81,15 & 90,21 & 78,91 & 75,48 \\
\hline \multicolumn{12}{|c|}{ Catimor } \\
\hline 1,83 & 14,96 & 0,89 & 11,76 & 0,58 & 12,46 & 0,74 & 100,00 & 100,00 & 100,00 & 86,83 & 83,29 \\
\hline 1,56 & 14,68 & 0,81 & 10,26 & 0,53 & 12,03 & 0,52 & 98,13 & 87,24 & 96,55 & 83,05 & 81,95 \\
\hline 1,25 & 14,57 & 0,81 & 10,07 & 0,48 & 11,72 & 0,38 & 97,39 & 85,63 & 94,06 & 82,23 & 80,44 \\
\hline 0,98 & 14,40 & 0,73 & 9,57 & 0,35 & 11,42 & 0,40 & 96,26 & 81,38 & 91,65 & 80,78 & 79,31 \\
\hline 0,79 & 14,24 & 0,73 & 9,41 & 0,37 & 10,90 & 0,47 & 95,19 & 80,02 & 87,48 & 79,68 & 76,54 \\
\hline 0,64 & 13,86 & 0,63 & 9,37 & 0,32 & 11,18 & 0,30 & 92,65 & 79,68 & 89,73 & 81,70 & 80,66 \\
\hline 0,41 & 13,85 & 0,71 & 9,37 & 0,63 & 11,16 & 0,56 & 92,58 & 79,68 & 89,57 & 81,69 & 80,58 \\
\hline 0,25 & 14,32 & 0,78 & 9,26 & 0,43 & 10,89 & 0,41 & 95,72 & 78,74 & 87,40 & 78,93 & 76,05 \\
\hline 0,21 & 14,42 & 0,78 & 9,28 & 0,32 & 10,81 & 0,30 & 96,39 & 78,91 & 86,76 & 78,43 & 74,97 \\
\hline 0,13 & 14,39 & 0,78 & 9,26 & 0,33 & 10,77 & 0,32 & 96,19 & 78,74 & 86,44 & 78,39 & 74,84 \\
\hline \multicolumn{12}{|c|}{ Conilon } \\
\hline 1,38 & 12,81 & 0,65 & 10,17 & 0,70 & 11,85 & 1,06 & 100,00 & 100,00 & 100,00 & 90,22 & 92,51 \\
\hline 1,15 & 12,15 & 0,69 & 9,55 & 0,54 & 10,86 & 1,27 & 94,85 & 93,90 & 91,65 & 88,90 & 89,38 \\
\hline 0,90 & 12,14 & 0,66 & 9,05 & 0,64 & 10,43 & 1,26 & 94,77 & 88,99 & 88,02 & 86,20 & 85,91 \\
\hline 0,64 & 12,31 & 0,76 & 8,73 & 0,37 & 10,30 & 1,24 & 96,10 & 85,84 & 86,92 & 84,03 & 83,67 \\
\hline 0,37 & 12,31 & 0,76 & 8,36 & 0,24 & 10,27 & 1,30 & 96,10 & 82,20 & 86,67 & 82,75 & 83,43 \\
\hline 0,34 & 12,33 & 0,73 & 8,33 & 0,45 & 10,18 & 1,25 & 96,25 & 81,91 & 85,91 & 82,32 & 82,56 \\
\hline 0,24 & 12,13 & 0,75 & 8,44 & 0,44 & 10,15 & 1,20 & 94,69 & 82,99 & 85,65 & 83,50 & 83,68 \\
\hline 0,21 & 12,17 & 0,76 & 8,40 & 0,42 & 10,12 & 1,29 & 95,00 & 82,60 & 85,40 & 83,10 & 83,16 \\
\hline 0,17 & 12,02 & 0,82 & 8,45 & 0,36 & 10,12 & 0,33 & 93,83 & 83,09 & 85,40 & 83,96 & 84,19 \\
\hline 0,12 & 12,04 & 0,81 & 8,42 & 0,35 & 10,19 & 0,35 & 93,99 & 82,79 & 85,99 & 83,96 & 84,63 \\
\hline
\end{tabular}

Na Tabela 1 observa-se a redução das dimensões ortogonais do fruto do café com a diminuição do teor de umidade. Segundo McMinn \& Magee (1997), a explicação para tal fato estar nas modificações estruturais associadas às alterações celulares do produto, devido a retirada de água durante o processo de secagem. Verifica-se também na Tabela 1 que o eixo médio (b), independente da espécie e variedade analisada, foi o que mais redução sofreu durante o processo de secagem, quando comparado com os demais eixos ortogonais. Tal fato sugere que a medida que ocorre o processo de desidratação, o fruto 
do café se afasta da forma esférica e aproxima-se da geometria de um esferóide oblato como também indicado, claramente pelos resultados dos valores de esfericidade e circularidade. O modelo matemático adequado para a descrição da relação das variáveis estudadas em função do teor de umidade dos frutos é da forma:

$$
Y=\beta+\beta_{1} X+\beta_{2} X^{2}
$$

em que:

Y - propriedades físicas esfericidade e circularidade, $\%$

$\mathrm{X}$ - teor de umidade, decimal base seca

$\beta, \beta_{1}, \beta_{2}$ - coeficientes da regressão

Na Tabela 2 são apresentados os valores estimados dos parâmetros $\beta, \beta_{1}$ e $\beta_{2}$ do modelo matemático selecionado para descrever as propriedades físicas estudadas.

Tabela 2. Parâmetros do modelo matemático para descrição da variação da esfericidade (E) e circularidade (C) dos frutos de café em função do teor de umidade

\begin{tabular}{|c|c|c|c|c|}
\hline \multirow{2}{*}{$\begin{array}{l}\text { Propriedade } \\
\text { Física }\end{array}$} & \multicolumn{3}{|c|}{ Parâmetros do Modelo } & \multirow{2}{*}{$\mathrm{R}^{2}$} \\
\hline & $\beta$ & $\beta_{1}$ & $\beta_{2}$ & \\
\hline \multicolumn{5}{|c|}{ Catuaí Vermelho } \\
\hline E & 81,785 & 1,263 & 1,240 & 0,9374 \\
\hline $\mathrm{C}$ & 77,691 & 4,636 & 0,421 & 0,8910 \\
\hline \multicolumn{5}{|c|}{ Catuaí Amarelo } \\
\hline $\mathrm{E}$ & 82,579 & 0,734 & 0,495 & 0,7205 \\
\hline $\mathrm{C}$ & 78,103 & 0,978 & 0,597 & 0,6927 \\
\hline \multicolumn{5}{|c|}{ Mundo Novo } \\
\hline $\mathrm{E}$ & 77,637 & 8,305 & $-2,007$ & 0,9482 \\
\hline $\mathrm{C}$ & 73,958 & 10,322 & $-3,732$ & 0,8934 \\
\hline \multicolumn{5}{|c|}{ Catimor } \\
\hline $\mathrm{E}$ & 78,962 & 0,813 & 1,613 & 0,8081 \\
\hline $\mathrm{C}$ & 74,999 & 5,818 & $-0,850$ & 0,6860 \\
\hline \multicolumn{5}{|c|}{ Conilon } \\
\hline $\mathrm{E}$ & 83,775 & $-3,444$ & 6,227 & 0,9466 \\
\hline $\mathrm{C}$ & 85,019 & $-8,522$ & 10,303 & 0,9807 \\
\hline
\end{tabular}

Na Figura 2 encontram-se os valores calculados e estimados dos índices de esfericidade e circularidade dos fruto do café, em função do teor de umidade, para as diferentes variedades estudadas. De modo geral, verifica-se nestas figuras a redução dos valores das propriedades físicas esfericidade e circularidade devido às alterações não homogêneas das características dimensionais do fruto do café com a diminuição do teor de umidade para todas as variedades analisadas. Entretanto, notase que entre as variedades estudadas a geometria do fruto do café Conilon foi a que mais se aproximou da forma esférica antes e durante o processo de secagem, apresentando menores variações de suas dimensões características, conforme a Tabela 1, o que resultou em menores reduções dos valores das propriedades esfericidade e circularidade dos frutos. Uma provável explicação para tal fato seria a reduzida quantidade de mucilagem presente nos frutos dessa espécie quando comparados com os das outras variedades analisadas da espécie arabica, uma vez ser esse constituinte rico em água e representar uma significativa parcela do volume do produto.
A.

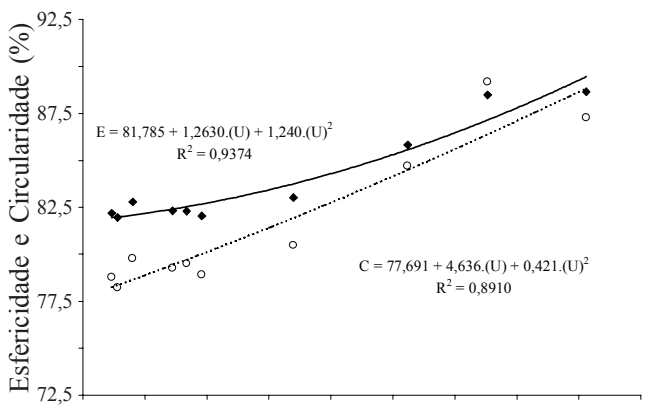

B.

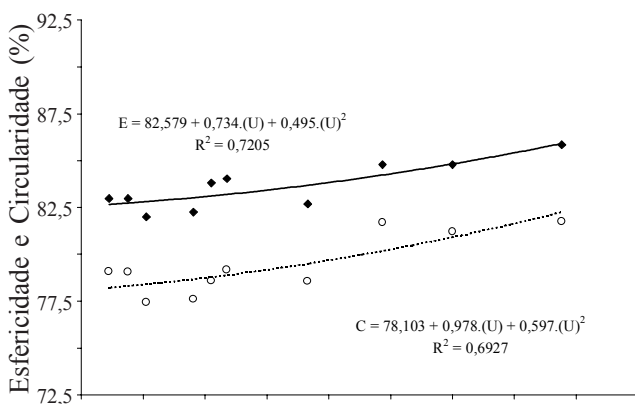

C.

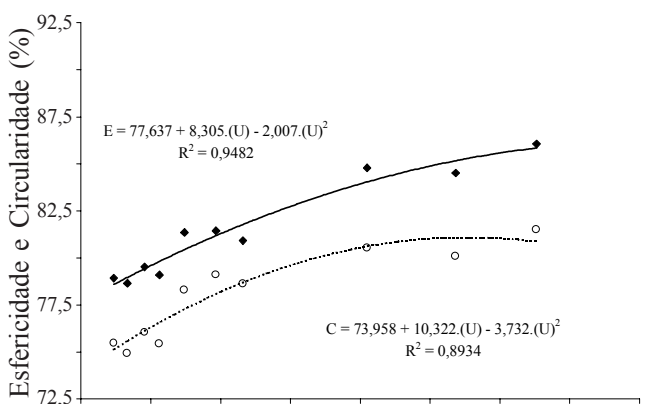

D.

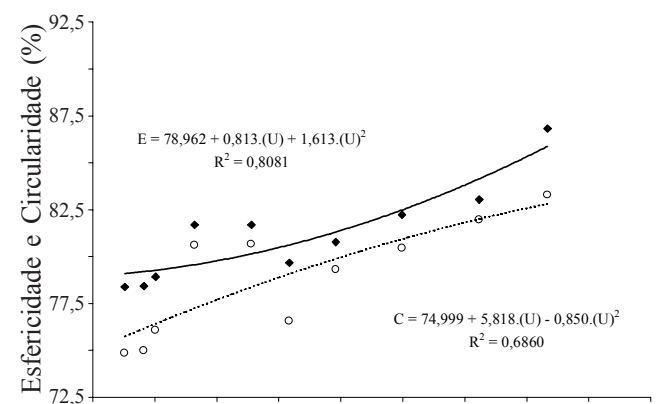

E.

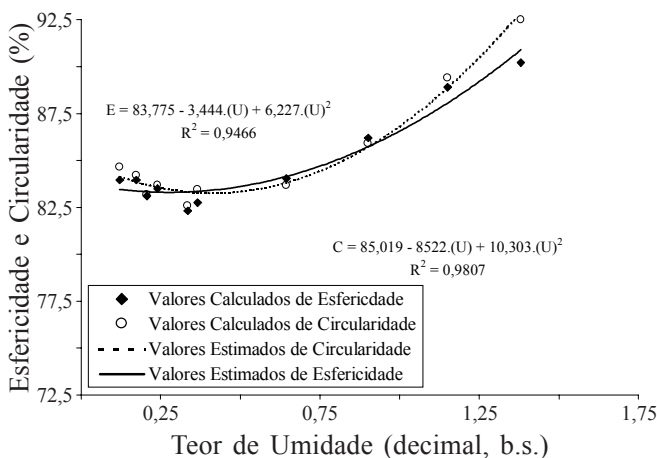

Figura 2. Valores calculados e estimados de esfericidade e circularidade dos frutos do café, em função do teor de umidade (U) para as variedades Catuaí Vermelho (A), Catuaí Amarela (B), Mundo Novo (C), Catimor (D) e Conilon (E) 
De modo geral, na Tabela 1 e nas Figuras 2 (A, B, C, D e E) verificam-se variações consideráveis da forma e das características dimensionais dos frutos do café das diferentes variedades durante o processo de secagem, o que vem confirmar que as variações das dimensões do produto durante sua desidratação, não devem ser negligenciadas nos diversos estudos associados aos processos de transferências de energia e massa que ocorrem ao longo da etapa de secagem. Verificase, ainda nas Figuras 2 (A, B, C, D e E), que as propriedades físicas dos frutos do café estudadas apresentam significativas reduções, quando comparadas com suas características iniciais. Sendo essa alteração mais acentuada para as variedades Catuaí Vermelho e Catuaí Amarelo.

\section{CONCLUSÕES}

1. Considerando-se o fruto do café um esferóide oblato, a redução do teor de umidade afetou as dimensões características do produto, provocando redução dos valores dos três eixos ortogonais, para todas variedades estudadas, indicando não serem desprezíveis as variações das dimensões do fruto, ao longo do processo de secagem.

2. A dimensão que mais sofreu alteração com a redução do teor de umidade foi o eixo ortogonal médio (b), para todas variedades analisadas.

3. A esfericidade e a circularidade do fruto diminuíram com a perda de água dos mesmos, indicando ser a geometria de um esferóide oblato mais adequada para representar a forma do fruto do café mais seco.

4. A geometria do fruto do café Conilon foi a que mais se aproximou da forma esférica, antes e durante o processo de secagem, apresentando menores variações de suas dimensões características; enquanto os frutos das variedades Catuaí Vermelho e Catuaí Amarelo foram os que apresentaram as maiores modificações na sua forma e dimensões.

\section{AGRADECIMENTOS}

Os autores agradecem à FINEP/RECOPE, EMPRAPA (PNP\&D - CAFÉ) e FAPEMIG pelo auxílio financeiro.

\section{LITERATURA CITADA}

Agrawal, K.K.; Clary, B.L.; Schroeder, E.W. Mathematical models of peanut pod geometry. ASAE, St. Joseph, 1972, 30p. Paper n ${ }^{\circ} 72-315$

Almeida, B.V. Determinação das propriedades físicas de amêndoas de cacau. Viçosa: UFV, 1979. 70p. Dissertação Mestrado

Bala, B.K.; Woods, J.L. Simulation of deep bed malt drying. Journal Agricultural Engineering Research, New York, v.30, n.3, p.235-244, 1984.

Brasil, Ministério da Agricultura e Reforma Agrária. Regras para análise de sementes. Brasília: DNDV/CLAV, 1992.365p.

Cavalcanti Mata, M.E.R.M.; Aragão, R.F.; Santana, E.F.; Silva, F.A.S. Estudo da morfologia geométrica em grãos. Revista Nordestina de Armazenagem, Campina Grande, v.3, n.1, p.3-30, 1986.

Fortes, M.; Okos, M.R. Changes in physical properties of corn during drying. Transaction of the ASAE, St. Joseph, v.23, n.4, p.1004-1008, 1980.

McMinn, W.A.M.; Magee, T.R.A. Physical characteristics of dehydrated potatoes - Part I. Journal of Food Engineering, London, v.33, n.1-2, p.37-48, 1997.

Mohsenin, N.N. Physical properties of plant and animal materials. New York: Gordon and Breach Publishers, 1986. 841p.

Ratti, C. Shrinkage during drying of foodstuffs. Journal of Food Engineering, London, v.23, n.1, p.91-105, 1994.

Soares, J.B. Curvas de secagem em camada fina e propriedades físicas de soja (Glicine max L.). Viçosa: UFV, 1986. 91p. Dissertação Mestrado

Sokhansanj, S.; Lang, W. Prediction of kernel and bulk volume of wheat and canola during adsorpsion and desorption. Journal Agricultural Engineering Research, New York, v.63, n.2, p.129-136, 1996.

Weber, E.A. Armazenagem agrícola. Porto Alegre: Gráfica e Editora La Salle, 1995. 395p.

Zogzas, N.P.; Maroulis, Z.B.; Kouris, D.M. Densities, shrinkage and porosity of some vegetables during air drying. Drying Technology, New York, v.12, n.7, p.1653-1666, 1994. 